\title{
Pressure Effects on the Segmental Dynamics of Hydrogen-Bonded Polymer Blends
}

\author{
S. H. Zhang, ${ }^{\dagger}$ R. Casalini, ${ }^{\ddagger, \S}$ J . Runt, ${ }^{\dagger}$ and C. M. Roland*, \\ Department of Materials Science and Engineering and Materials Research Institute, \\ The Pennsylvania State University, University Park, Pennsylvania 16802; Chemistry Division, \\ Code 6120, Naval Research Laboratory, Washington D.C. 20375-5342; and Chemistry Department, \\ George Mason University, Fairfax, Virginia 22030
}

Received August 15, 2003; Revised Manuscript Received October 23, 2003

\begin{abstract}
Segmental relaxations in the neat components and the blend of poly(4-vinylphenol) (PVPh) with poly(ethylene-co-vinyl acetate) (EVA, with 70 wt \% vinyl acetate) are studied by broadband dielectric spectroscopy at different temperatures and hydrostatic pressures (up to $750 \mathrm{MPa}$ ). Pressure retards the relaxation, with a consequent increase of the glass transition temperature $\left(T_{g}\right)$. The pressure coefficient of $\mathrm{T}_{\mathrm{g}}$ is $158 \mathrm{C} / \mathrm{GPa}$ for the neat EVA, with temperature and pressure found to exert a comparable effect on segmental relaxation. The shape of the EVA segmental relaxation function, however, is the same for different temperature-pressure conditions at a given relaxation time. On the other hand, PVT measurements on PVPh indicate that temperature is more important than pressure in determining the segmental relaxation time, due to the presence of strong hydrogen bonding. For blends with $20 \%$ and $30 \%$ PVPh, similar pressure-dependent increases of $\mathrm{T}_{\mathrm{g}}$ are observed, al though contributions from $\mathrm{T}$ and $\mathrm{P}$ to segmental relaxation are again comparable, despite the hydrogen bonding between PVPh and EVA. Although both high pressure and temperature reduce the concentration of hydrogen bonds in the bl ends, which tends to decouple the components' segmental relaxation processes, the relaxation time distribution in the blends is narrowed with increasing pressure at a given relaxation time. This behavior is interpreted by considering the additional mobility achieved from breaking hydrogen bonds at high pressures and high temperatures for the hydrogen-bonded (and thus slow) PVPh-EVA segments compared with the fast relaxation of unassociated EVA segments.
\end{abstract}

\section{Introduction}

As first shown by NMR, ${ }^{1}$ significant dynamic heterogeneity exists in miscible polymer blends having a large difference in components' gl ass transition temperatures $\left(\Delta \mathrm{T}_{\mathrm{g}}\right)$ and without strong intermolecular interactions. ${ }^{2}$ Specifically, two segmental processes ( $\alpha$ relaxations) can be observed in dielectric/mechanical relaxation spectra, and components' relaxation times are distinguishable, although a single $T_{g}$ is observed by DSC. This has been interpreted by considering different mechanisms, i.e., intrinsic mobility difference, concentration fluctuations, and, more recently, chain-connectivity effects. ${ }^{3-5}$

Whereas most studies are performed with temperature as the variable, the effect of pressure on the dynamic heterogeneity has recently been investigated. In a dielectric study on poly(isoprene-b-vinylethylene) diblock copolymer (PI-b-PVE), Floudas and co-workers found that high pressure can induce dynamic homogeneity. ${ }^{6}$ Although the two blocks relax individually at atmospheric pressure, giving rise to two $\alpha$ relaxations, the fast process (relatively weak and arising from the low- $\mathrm{T}_{\mathrm{g}} \mathrm{PI}$ ) shifts to low frequency and merges with the slow one when high hydrostatic pressure is applied. This was attributed to the stronger pressure dependence of the PI block compared to that of PVE. However, in another dielectric study on the blend of polystyrene with poly(vinyl methyl ether) (PS/PVME), in which the more pressure-sensitive material (PS) has also a much higher $\mathrm{T}_{\mathrm{g}},{ }^{7,8}$ Alegría et al. ${ }^{9}$ found that applied pressure has no effect beyond increasing the $T_{g}$ of the blend. The segmental relaxation time distributions were essentially

\footnotetext{
† The Pennsylvania State University.

₹ Naval Research Laboratory.

$\S$ George Mason University.

* Corresponding author.
}

identical at different temperature-pressure conditions, if their relaxation times were the same (i.e., isokinetic). The authors concluded that the influence of pressure on the component segmental dynamics was equivalent to that of temperature. ${ }^{9}$ It should be noted that in the second example only the PVME relaxation can be monitored dielectrically, since the PS repeat unit has a much smaller dipole moment than the PVME chain unit.

In this paper, we present a dielectric study on the blend of poly(4-vinyl phenol) (PVPh) with poly(ethyleneco-vinyl acetate) (EVA, 70 wt \% vinyl acetate) using both pressure and temperature as experimental variables. The strong intermolecular hydrogen bonding between hydroxyl groups of PVPh and carbonyl groups of EVA is responsible for the miscibility, ${ }^{10}$ and it also makes the blend more dynamic homogeneous by coupling the components' segmental relaxations. ${ }^{11}$ Although $\Delta T_{g}$ between PVPh and EVA is much larger than that between the components in PI/PVE and PS/PVME blends, our previous atmospheric pressure study found that dynamic homogeneity can be achieved at appropriate compositions (i.e., at PVPh concentrations of 30 and 40 wt \%), for which intercomponent, rather than intramolecular, interactions dominate, whereas two $\alpha$ processes are observed in blends containing 20\% PVPh. The latter observation was rationalized by considering the coexistence of both hydrogen-bonded (slow process) and unassociated ("free") EVA segments (fast process) as a consequence of the stoichiometry of $\mathrm{H}$-bond formation. ${ }^{11,12}$

The dielectric relaxation in PVPh/EVA blends includes contributions from both EVA and PVPh, since they have comparable dipole moments. ${ }^{13}$ The current study is also motivated by the observation that el evated hydrostatic pressure reduces and weakens hydrogen bonding in low molecular weight alcohols. ${ }^{14-19}$ Conse- 
quently, we are interested in how pressure will influence the segmental dynamics in associated polymer blends. The relative contributions of temperature and volume to the $\alpha$ relaxations are al so eval uated, with a discussion of the implications concerning the mechanism for dynamic heterogeneity.

\section{Experimental Section}

The EVA (Scientific Polymer Products, Inc.) had $\mathrm{M}_{\mathrm{w}}=$ 441000 and $M_{w} / M_{n}=7.2$, and for the PVPh (Aldrich, Inc.) $M_{w}=21000$ and $M_{w} / M_{n}=2.5$. Both polymers were used as received. $\mathrm{T}_{\mathrm{g}}$ of EVA and PVPh are -15 and $170{ }^{\circ} \mathrm{C}$, respectively, as measured by a Seiko SSC5200 DSC at a heating rate of $10^{\circ} \mathrm{C} / \mathrm{min}$. It should be noted that the PVPh in the present study is from a different lot, and has a different molecular weight, than the material used a previous study at atmospheric pressure. ${ }^{11}$ Although the EVA sample has substantial polydispersity, its molecular weight is very high, far above the entangl ement molecular weight, so that the molecular weight distribution is not significant herein.

Blends were prepared by mixing appropriate amounts of 5 wt \% solutions of the components in methyl ethyl ketone. The mixed solutions were cast onto Teflon-coated foil dishes, and the solvent was removed in a vacuum oven by gradually increasing temperature to $\sim 20$ deg above $T_{g}$ for at least 1 week. We will focus on blends with $20 \%$ and $30 \%$ PVPh (denoted as VA20 and VA30, respectively), which exhi bit dynamic heterogeneity and homogeneity, respectively, at atmospheric pressure.

Dielectric spectra were obtained with an IMASS time domain dielectric analyzer $\left(10^{-4}-10^{4} \mathrm{~Hz}\right)$ and a Novocontrol Alpha analyzer $\left(10^{-2}-10^{6} \mathrm{~Hz}\right)$. The sample film was contained between parallel plates, placed within a Manganin pressure cell (Harwood Engineering). The capacitor was isolated from the pressurizing fluid (a light hydrocarbon oil) by means of a Teflon ring and tape. Pressure was applied using an E nerpac hydraulic pump, in combination with a pressure intensifier (Harwood Engineering), and measured with a Sensotec tensometric transducer (resolution $=150 \mathrm{kPa}$ ). Temperature stability for all experiments was better than $\pm 0.1 \mathrm{deg}$.

Pressure-volume-temperature (PVT) measurements were carried out using a Gnomix instrument. The method is based on the confining fluid technique, with the sample surrounded by mercury. I sobaric volumes were measured during cooling at a rate of $0.1 \mathrm{deg} / \mathrm{min}$ at hydrostatic pressures from 10 to $200 \mathrm{MPa}$.

\section{Results}

Pressure Effects on the Segmental Relaxation of EVA. Applying pressure to EVA, similar to decreasing the temperature, shifts the segmental relaxation peak to lower frequency. However, the shape of the relaxation function is essentially identical to that at atmospheric pressure at a given relaxation time (Figure 1). Similar behavior has been observed for other neat polymers. ${ }^{7,20-24}$

The temperature dependence of the segmental relaxation time $\tau_{\alpha}$ (defined as $=\left(2 \pi \mathrm{f}_{\max }\right)^{-1}$, where $\mathrm{f}_{\max }$ is the frequency of the maximum in the dielectric loss) for polymers is typically non-Arrhenius and can be described by the Vogel-Fulcher (VF) equation. Since increasing pressure $(\mathrm{P})$ densifies the material, analogous to lowering temperature, a nonlinear dependence of log$\left(\tau_{\alpha}\right)$ on pressure is also expected and observed (Figure 2). The VF equation can be modified to describe the pressure dependence at constant temperature:25,26

$$
\tau_{\alpha}=\tau_{0} \exp \left(\frac{\mathrm{D}_{\mathrm{P}} \mathrm{P}}{\mathrm{P}_{0}-\mathrm{P}}\right)
$$

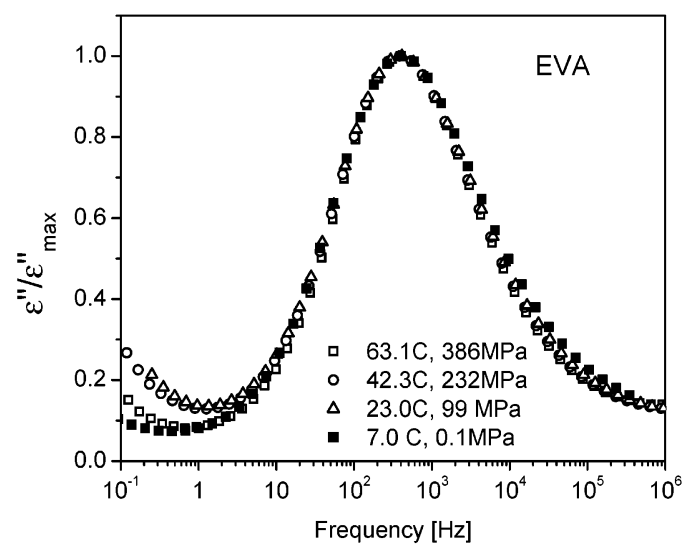

Figure 1. Dielectric loss spectra of EVA at different temperatures and pressures. For comparison purposes, $\epsilon^{\prime \prime}$ has been normalized by the corresponding $\epsilon^{\prime \prime}$ max and the frequency is shifted less than 1 decade to superpose the peaks. Specifically, the spectrum at $0.1 \mathrm{MPa}$ is unshifted, the spectra at 23 and $42.3^{\circ} \mathrm{C}$ are shifted of 0.18 and 0.21 decades, respectively, to lower frequency, and the $63.1{ }^{\circ} \mathrm{C}$ spectrum is shifted 0.02 decades higher in frequency.

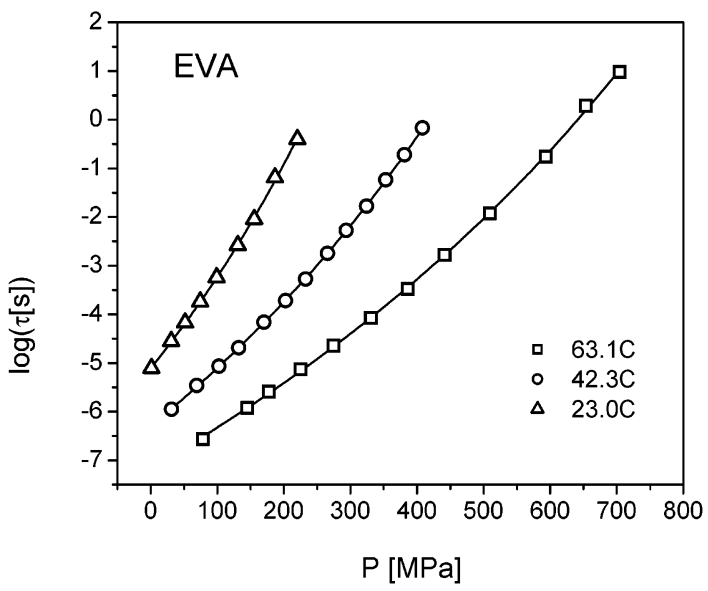

Figure 2. Pressure dependence of the segmental relaxation time $\tau_{\alpha}$ of EVA at different temperatures. Solid lines are best fits to eq 1 .

Table 1. Fit Parameters of Eq 1 to the Dielectric

Relaxation vs Pressure Data in Figures 2 and 8

\begin{tabular}{ccccc}
\hline sample & $\mathrm{T}\left[{ }^{\circ} \mathrm{C}\right]$ & $\log \left(\tau_{0}[\mathrm{~s}]\right)$ & $\mathrm{D}_{\mathrm{P}}{ }^{\mathrm{a}}$ & $\mathrm{P}_{0}[\mathrm{MPa}]$ \\
\hline EVA & 23.0 & $-5.10 \pm 0.02$ & $41 \pm 2$ & $1050 \pm 34$ \\
EVA & 42.3 & $-6.29 \pm 0.02$ & $41 \pm 2$ & $1600 \pm 47$ \\
EVA & 63.1 & $-7.16 \pm 0.02$ & $41 \pm 2$ & $2245 \pm 60$ \\
VA20 & 51.0 & $-5.88 \pm 0.03$ & $100 \pm 10$ & $2540 \pm 250$ \\
VA20 & 74.6 & $-7.22 \pm 0.04$ & $100 \pm 10$ & $3680 \pm 350$ \\
VA20 & 94.4 & $-7.91 \pm 0.05$ & $100 \pm 10$ & $4700 \pm 440$ \\
VA30 & 70.3 & $-5.00 \pm 0.07$ & $18.8 \pm 3$ & $630 \pm 60$ \\
VA30 & 90.8 & $-5.8 \pm 0.1$ & $18.8 \pm 3$ & $900 \pm 80$
\end{tabular}

a The parameter $D_{p}$ was determined to be a pressure-independent material constant.

in which $\tau_{0}, D_{P}$, and $P_{0}$ are fitting parameters. The best fit to eq 1 is reported as a line in Figure 2 for EVA and Figure 8 for the two blends, while the obtained parameters are in Table 1.

To quantify the effect of pressure on the segmental relaxation time, a reference temperature, $\mathrm{T}_{\alpha}$, is defined as the temperature at which $\tau_{\alpha}=1 \mathrm{~s}$. This can be regarded as the dynamic version of a calorimetric $T_{g}$. The value chosen for the reference $\tau_{\alpha}$ is arbitrary, and $1 \mathrm{~s}$ avoids data extrapolation. The reference temperature can be directly obtained from Figure 2, and its 


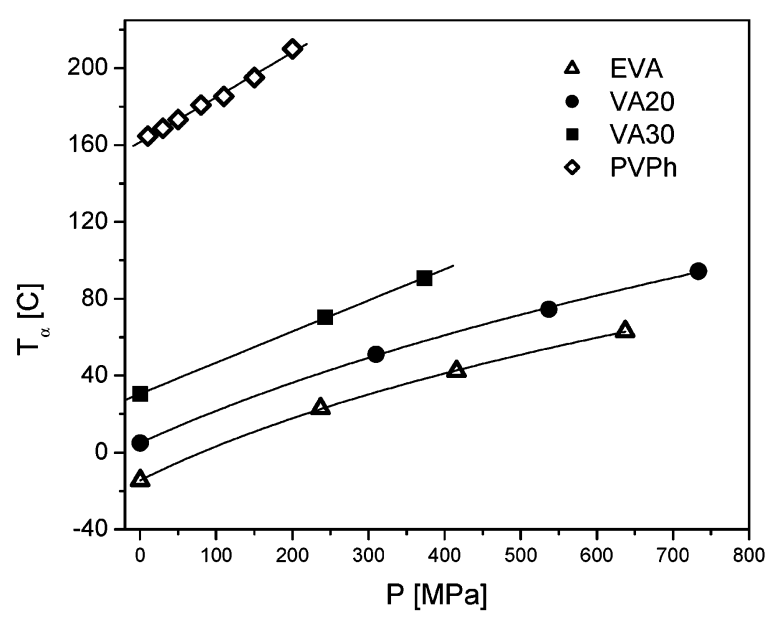

Figure 3. Pressure dependence of the reference temperature $\mathrm{T}_{\alpha}$. For PVPh, PVT data were used to extract $\mathrm{T}_{\mathrm{g}}$ 's at different pressures. For others, $\mathrm{T}_{\alpha}$ is defined as the temperature at which $\tau_{\alpha}=1 \mathrm{~s}$. Lines are drawn to guide the eyes.

pressure dependence is plotted in Figure 3. The pressure dependence of $T_{\alpha}$ deviates from linearity and can be described by the empirical Andersson equation ${ }^{27}$ (which can be also derived from a theoretical model ${ }^{23}$ ). Approaching atmospheric pressure, $\mathrm{dT}_{\alpha} / \mathrm{dP} \approx 0.158 \mathrm{C} / \mathrm{MPa}$. Note this is significantly smaller than the value for the structurally similar poly(vinyl acetate) (PVAc), for which $\mathrm{dT}_{\alpha} / \mathrm{dP} \approx 0.267 \mathrm{C} / \mathrm{MPa}$ (cal culated from the data in ref 23 for $\tau_{\alpha}=1 \mathrm{~s}$ and $\mathrm{P}=0.1 \mathrm{MPa}$ ).

Although the rapid increase of $\tau_{\alpha}$ for a polymer when approaching $\mathrm{T}_{\mathrm{g}}$ from above is well-known, the molecular mechanisms underlying this behavior remain in dispute. The classic free volume model invokes that densification during cooling or pressurization, gives rise to strong intermolecular cooperativity; that is, because of the lack of sufficient local free vol ume, the relaxation of one unit is only possible by cooperating with surrounding units. However, this idea cannot be assessed by conventional temperature-domain experiments, since temperature changes affect not only volume but also the available thermal energy.

By applying elevated hydrostatic pressure, recent studies have found that temperature is equival ently, if not more, important than pressure in governing relaxation times near the glass transition.7,19,22,23,28-30 To compare the relative contributions to the temperature dependence of $\tau$ from thermal energy and volume, Ferrer and co-workers proposed using the ratio of the thermal expansion coefficient at constant relaxation time, $\alpha_{\tau}=-\mathrm{V}\left(\partial \mathrm{V}^{-1} / \partial \mathrm{T}\right)_{\tau}$, to that at constant pressure, $\alpha_{\mathrm{P}}=-\mathrm{V}\left(\partial \mathrm{V}^{-1} / \partial \mathrm{T}\right)_{\mathrm{P}}$, where $\mathrm{V}$ is the specific volume. ${ }^{17}$ If the relaxation is controlled by volume, $\alpha_{\tau} /\left|\alpha_{P}\right|$ will be close to unity, whereas $\alpha_{\tau} /\left|\alpha_{p}\right| \gg 1$ for thermally activated dynamics.

Volumes at different conditions of $\mathrm{T}$ and $\mathrm{P}$ can be obtained from PVT measurements (Figure 4). Above $T_{g}$, the specific volume of amorphous EVA can be described by the Tait equation: ${ }^{31}$

$$
\mathrm{V}(\mathrm{T}, \mathrm{P})=\mathrm{V}(\mathrm{T}, 0)\{1-0.0894 \ln [1+\mathrm{P} / \mathrm{B}(\mathrm{T})]\}
$$

in which $V(T, 0)$, the specific volume (in $\mathrm{mL} / \mathrm{g}$ ) at $\mathrm{P}=0$, is usually approximated by the value at atmospheric pressure. For EVA, V $(T, 0)=0.93828+5.5785 \times 10^{-4} \mathrm{~T}$ $+3.6206 \times 10^{-7} \mathrm{~T}^{2}$, and the pressure coefficient $\mathrm{B}(\mathrm{T})=$

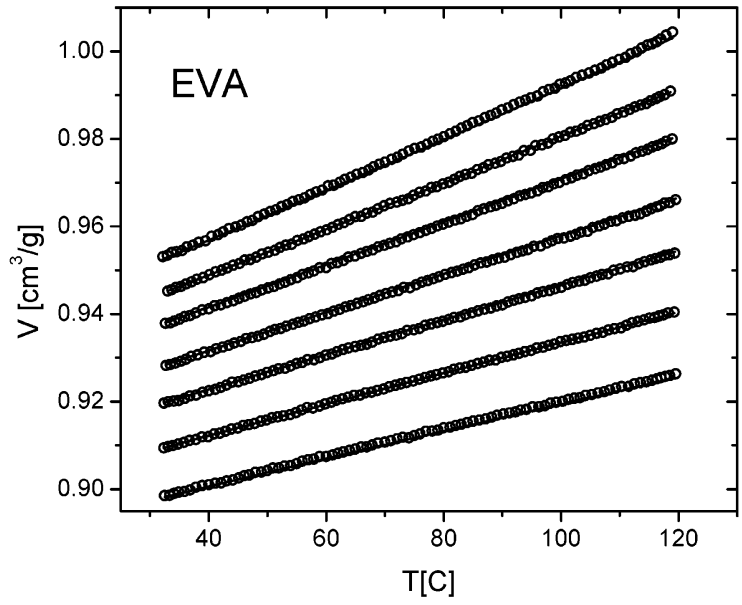

Figure 4. PVT data of EVA measured at a cooling rate of 0.5 $\mathrm{C} / \mathrm{min}$. Lines are the best fit to the Tait equation (eq 2). The vertical axis represents the specific volume. The pressures were (from top to bottom) 10,30,50,80, 110, 150, and 200 $\mathrm{MPa}$.

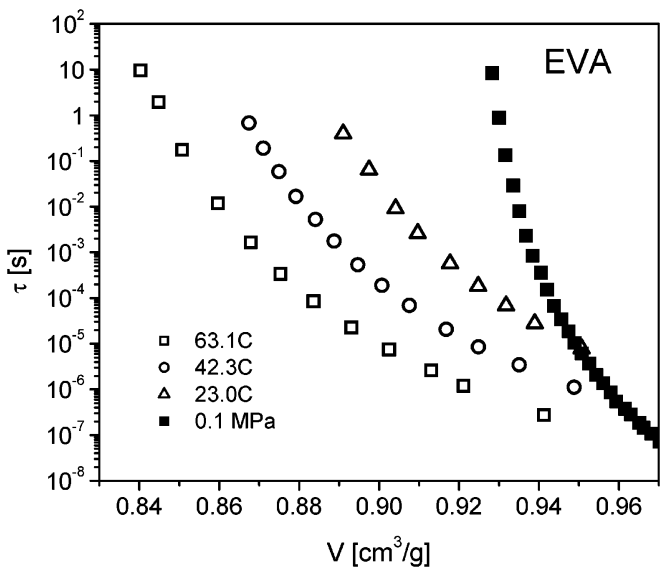

Figure 5. Dependence of $\alpha$ relaxation time on the specific volume of EVA. The data are converted from Figure 2 with the aid of PVT data (F igure 4). Solid squares are isobaric data measured at $\mathrm{P}=0.1 \mathrm{MPa}$ as a function of temperature.

$241 \exp \left(-5.273 \times 10^{-3} \mathrm{~T}\right)$. The units for $\mathrm{T}$ and $\mathrm{P}$ are ${ }^{\circ} \mathrm{C}$ and $\mathrm{MPa}$, respectively.

Whereas $\alpha_{p}$ can be calculated directly the from PVT data, calculation of $\alpha_{\tau}$ requires the $T$ and $V$ values corresponding to a specified $\tau_{\alpha}$. Using the Tait equation, pressure (in Figure 2) and temperature dependences (data not shown) are converted to the vol ume dependences displayed in Figure 5. In Figure 6 we plot, as a function of temperature, the specific volumes for constant (atmospheric) pressure and for $\tau_{\alpha}=1 \mathrm{~s}$. From the slopes of these two lines, we obtain $\alpha_{\tau} /\left|\alpha_{P}\right|=2.1$, which suggests that both temperature and vol ume contribute significantly to the temperature dependence of the relaxation times of EVA. This result is close to that for PVAc, for which $\alpha_{\tau} /\left|\alpha_{\mathrm{P}}\right|=1.8$ at $\tau_{\alpha}=100 \mathrm{s.}^{23,24}$ (Note that the expansivity ratio is relatively insensitive to the particular value of $\tau_{\alpha}$ used.)

For PVPh, because of strong dc conductivity, the segmental relaxation process could not be resolved, precluding a direct determination of $\alpha_{\tau} /\left|\alpha_{p}\right|$. However, the ratio $E_{V} / E_{P}$ can be used for the same purpose, in which $E_{v}$ and $E_{p}$ are the apparent activation energies respectively at constant volume (isochoric) and constant pressure (isobaric). Since $E_{v}$ includes contribution only from thermal energy and $E_{p}$ from both volume and 


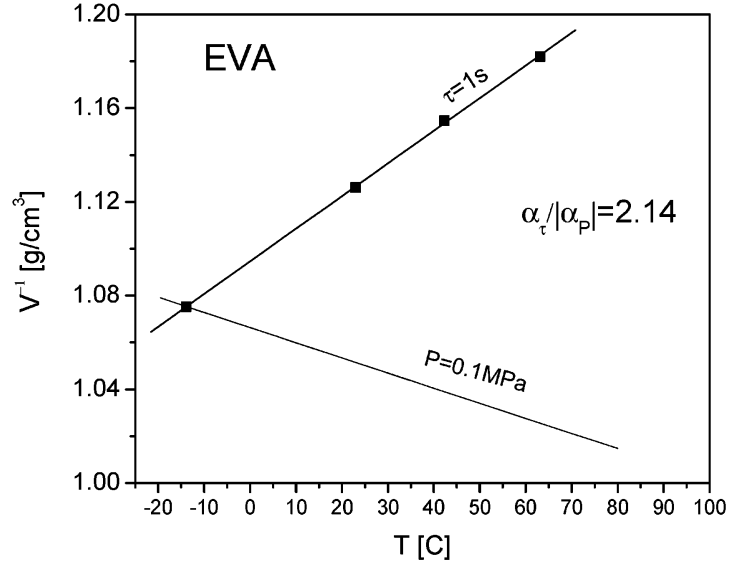

Figure 6. Densities of EVA at constant relaxation time $\left(\tau_{\alpha}=\right.$ $1 \mathrm{~s})$ and at constant pressure (0.1 MPa). From the ratio of their slopes, $\alpha_{\tau} /\left|\alpha_{P}\right|=2.14$ is obtained.

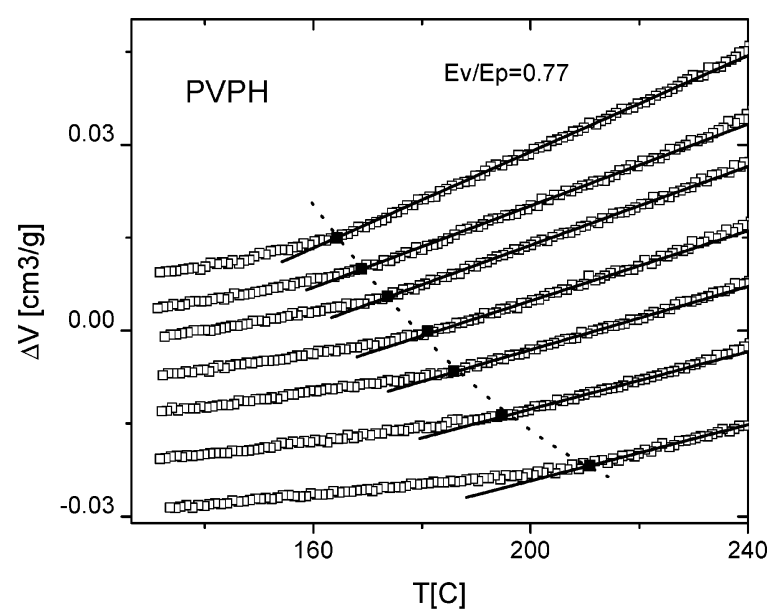

Figure 7. PVT measurements of PVPh at a cooling rate of $0.5 \mathrm{C} / \mathrm{min}$. The solid squares refer to the $\mathrm{T}_{\mathrm{g}}$ at different pressures. The pressures were (from top to bottom) 10, 30, 50, $80,110,150$, and $200 \mathrm{MPa}$

thermal energy, a value of $E_{v} / E_{p}=1$ suggests that the segmental relaxation is simply a thermally activated process, whereas a ratio approaching zero indicates that volume controls the $T$ dependence of the relaxation times. Although $\mathrm{E}_{\mathrm{V}}\left(=\mathrm{R}\left(\partial \ln \tau / \partial \mathrm{T}^{-1}\right) \mid \mathrm{v}\right)$ and $\mathrm{E}_{\mathrm{P}}(=\mathrm{R}(\partial$ In $\left.\tau / \partial T^{-1}\right)\left.\right|_{\mathrm{P}}$ ) can in principle be obtained from dielectric spectra measured at different temperatures and pressures, herein we calculate their ratio for PVPh from the relation ${ }^{32}$

$$
\frac{\mathrm{E}_{\mathrm{V}}}{\mathrm{E}_{\mathrm{P}}}=1-\gamma\left(\frac{\partial \mathrm{T}}{\partial \mathrm{P}}\right)_{\tau}
$$

In eq $3,(\partial T / \partial P)_{\tau}$ is just $d T_{g} / d P .{ }^{33}$ By defining $T_{g}$ as the temperature at which the thermal expansion coefficient abruptly changes (Figure 7), we obtain $\mathrm{dT} / \mathrm{dP}=0.22$ $\pm 0.02 \mathrm{C} / \mathrm{MPa}$. From the PVT data, the thermal pressure coefficient, $\gamma \equiv(\partial \mathrm{P} / \partial \mathrm{T})_{\mathrm{V}}$, is $1.034 \mathrm{MPa} / \mathrm{C}$. This leads to $E_{V} / E_{P}=0.77$ for neat PVPh. Using the relation ${ }^{8}$

$$
\frac{E_{V}}{E_{P}}=\frac{1}{1-\alpha_{\mathrm{P}} / \alpha_{\tau}}
$$

we calculate $\alpha_{\tau} /\left|\alpha_{P}\right|=3.3$. This suggests that, in comparison to the EVA, temperature plays a more important role than pressure in the segmental relax-
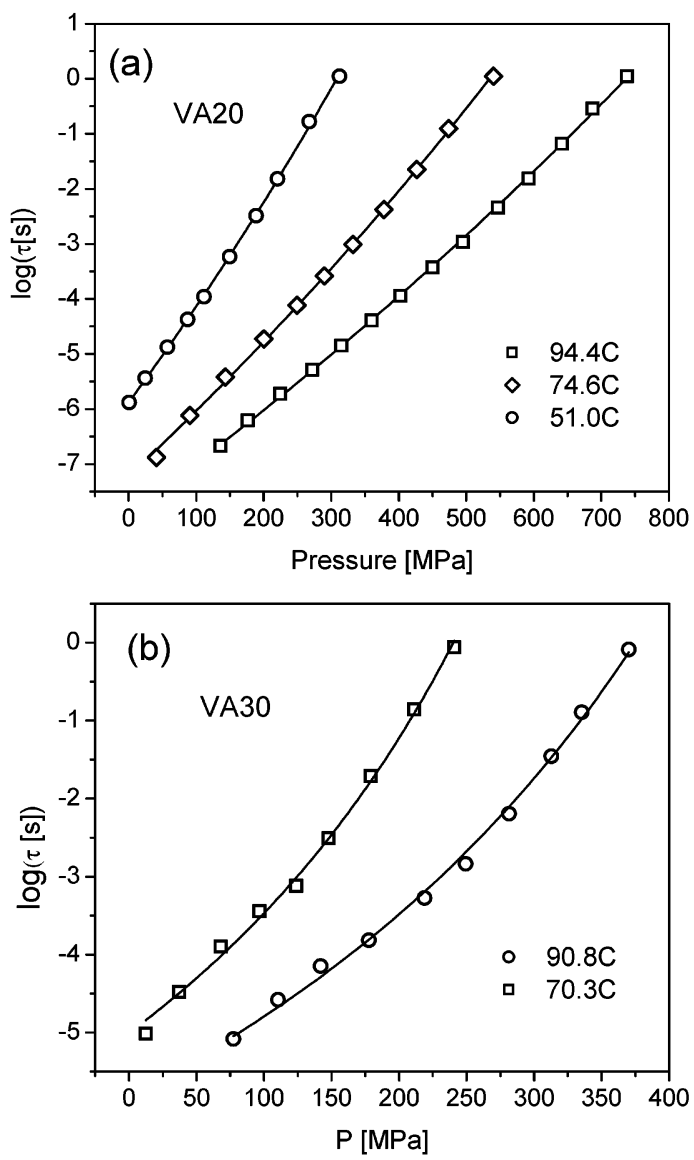

Figure 8. Pressure dependence of the segmental relaxation time at different temperatures: (a) VA20 and (b) VA30. The solid lines are the best fits to eq 1; the obtained parameters are in Table 1.

ation of PVPh. This is expected given the latter's strong intramolecular hydrogen bonding. The $\alpha_{\tau} /\left|\alpha_{P}\right|$ value in PVPh is consistent with those of low molecular weight associated liquids; e.g., $\alpha_{\tau} /\left|\alpha_{P}\right|=6$ for sorbitol ${ }^{18}$ and 8 for propylene glycol..$^{8}$

Pressure Effects in Blends. In general, segmental relaxation times in the miscible blends with $20 \%$ and $30 \%$ PVPh have pressure dependences similar to that for the neat EVA. From the variation of $\tau_{\alpha}$ with pressure (Figure 8), we find $\mathrm{dT}_{\alpha} / \mathrm{dP}=0.15 \pm 0.02$ and $0.16 \pm$ $0.02 \mathrm{C} / \mathrm{MPa}$ for VA20 and VA30, respectively (Figure $3)$, close to that of neat EVA. $\alpha_{\tau} /\left|\alpha_{p}\right|$, calculated as described above, equals 2.1 and 2.2 for VA20 and VA30, respectively. The values, reflecting a comparable influence of temperature and pressure on the relaxation times, are significantly smaller than for PVPh, presumably due to less intermolecular hydrogen bonding.

Figure 9 shows the dielectric spectra in the region of the segmental relaxation for VA20 at various el evated pressures. With increasing pressure there is a decrease of the ionic conductivity and an increase of the relaxation time, which is analogous to the behavior observed in other materials. ${ }^{8,34,35}$ Careful analysis of the data demonstrates that dynamic heterogeneity still exists in this blend at high pressure, although the relaxation becomes narrower compared with that at atmospheric pressure. This is seen more clearly in Figure 10, which shows the spectra at different $(T, P)$ conditions for a fixed value of the relaxation time. Whereas the full width at half-maximum (fwhm) is still quite large ( $\sim 6$ decades), a decrease of $\sim 1$ decade is clearly observed 


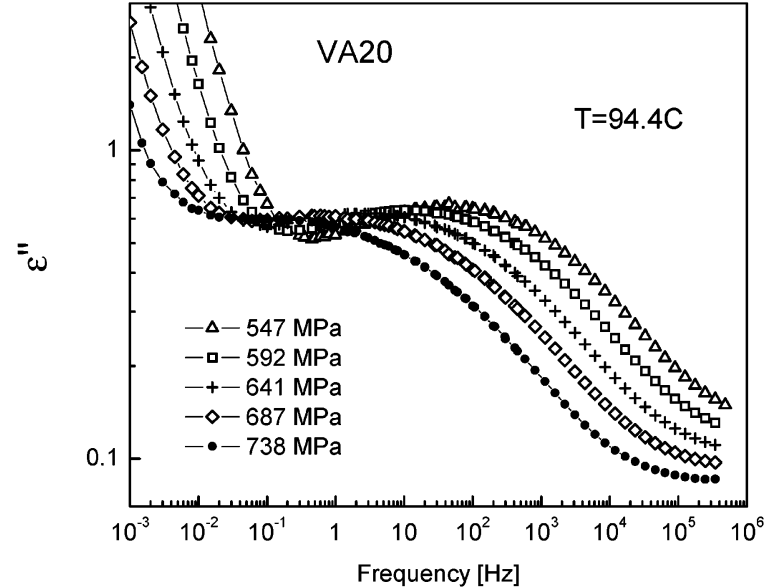

Figure 9. Dielectric loss spectra of VA20 at various el evated pressures.
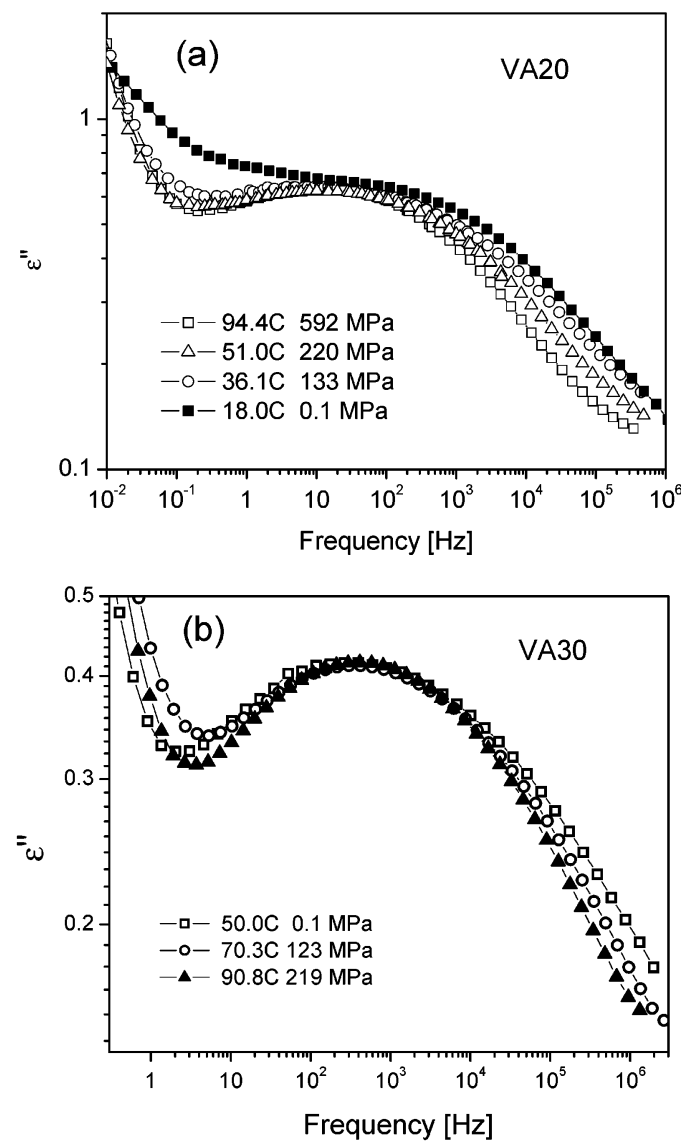

Figure 10. Comparison of dielectric $\alpha$ relaxations at different temperatures and pressures at a fixed relaxation time: (a) VA20 and (b) VA30.

when pressure is increased from 133 to $592 \mathrm{MPa}$ for VA20. (The corresponding temperature change is from 36.0 to $94.4{ }^{\circ} \mathrm{C}$.) That is, the blends have become dynamically more homogeneous with increasing pressure. Nonetheless, the shoulder on the low-frequency side of the relaxation is still visible even at the highest pressure, indicating that two types of relaxing segments (intermolecular hydrogen-bonded and unassociated EVA) are still relaxing at distinctly different rates. The pressure-induced dynamic homogeneity, clearly observed in PI-b-PVE at lower pressure ( $315 \mathrm{MPa}),{ }^{6}$ is not found for VA20. The narrowing of the relaxation time distribution in VA30 is similar to that in VA20
(Figure 10b).

\section{Discussion}

The influence of pressure on $\tau_{\alpha}$, and the comparable contribution from temperature and pressure, is consistent with other studies on polymers and blends. However, the narrower relaxation time distribution in PVPh/ EVA blends at el evated pressure is rather unexpected, considering that the hydrogen bond fraction should be lower at higher (T or P). ${ }^{14-19}$ Whereas the decrease of hydrogen-bonded fraction with increasing temperature is a well-established concept, ${ }^{10}$ high pressure al so leads to a reduction in hydrogen bonding due to the increased geometric hindrance, which requires not only a suitable distance between corresponding functional groups but also specific relative orientations. The decrease in intermolecular hydrogen bonds increases the fraction of unassociated EVA segments, serving to exaggerate the dynamic heterogeneity. The relaxation time distribution should be broader at high pressure if the change in hydrogen bond fraction is the dominant influence. Further reduction in intermolecular associations can decouple the components' segmental relaxation processes. For example, we observed a single dielectric segmental relaxation process in $\mathrm{VA} 30$ at $\mathrm{P}=0.1 \mathrm{MPa}$, whose intermolecular hydrogen bonding fraction (per EVA) is only slightly higher than VA20.11 If the above argument is correct, one would anticipate two $\alpha$ relaxations in VA30 at high pressure. The fact that the opposite result is observed indicated that other mechanisms need to be considered, in particular the relative change in segmental mobilities.

To reiterate, two different relaxing segments were observed in our previous study of VA20 at atmospheric pressure: hydrogen-bonded PVPh-EVA (slow relaxation) and unassociated EVA (fast relaxation). ${ }^{11}$ These may also exist in VA30, but the fraction of "free" EVA segments is very low and their relaxation is masked by the dominant slow process. High temperature affects the non-hydrogen-bonded EVA segments by simply enhancing their mobility (via increased thermal energy and free volume), whereas high pressure decreases their mobility through densification. These two competing factors are almost equally important, as implied by the value of $\alpha_{\tau} /\left|\alpha_{p}\right|=2.1$ for neat EVA. However, for hydrogen-bonded segments, additional mechanisms are involved. Both high temperature and pressure weaken and reduce hydrogen bonding, thus influencing the relaxation of hydrogen-bonded PVPh-EVA segments in the same fashion (shifting the slow process to higher frequency, closer to the fast process). The net result of high pressure/high temperature is therefore a reduction in the difference between the relaxation times of the two segments and a consequent narrowing of the relaxation time distribution.

Other possi ble mechanisms can also be considered in interpreting the narrowing of the segmental dispersion with pressure. Floudas and co-workers ${ }^{6}$ have proposed that a larger activation volume, $\mathrm{V}^{\#}$, of the low- $\mathrm{T}_{\mathrm{g}} \mathrm{PI}$ block in PI-b-PVE reduces the dynamic heterogeneity and gives rise to the homogeneity at sufficiently high hydrostatic pressure in that material. The activation volume is defined $a^{36}$

$$
\mathrm{V}^{\#}=\mathrm{RT} \frac{\mathrm{d} \ln \left(\tau_{\alpha}\right)}{\mathrm{dP}}=\mathrm{m} \frac{\mathrm{dT}}{\mathrm{dP}} \mathrm{R} \ln (10)
$$




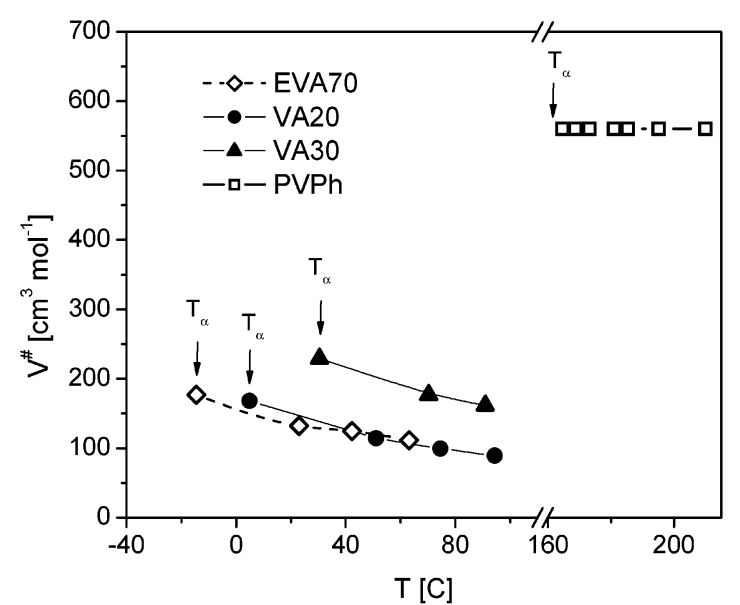

Figure 11. Activation volume of neat polymers and blends at different temperatures. Lines are drawn to guide the eyes.

where $m=d \log \left(\tau_{\alpha}\right) /\left.d\left(T_{\alpha} / T\right)\right|_{T=T_{\alpha}}$ is the so-called fragility, a parameter used to characterize the rapidity at which $\tau_{\alpha}$ changes across $\mathrm{T}_{\alpha} \cdot{ }^{37} \mathrm{~F}$ or neat EVA, $\mathrm{V}^{\#}$ can be determined directly from Figure 2, with the results provided in Figure 11 . $\mathrm{V}^{\#}$ exhibits the customary decrease with temperature and increase with pressure. At $\mathrm{P}=0.1 \mathrm{MPa}, \mathrm{V}^{\#}$ for EVA changes from $\sim 170 \mathrm{~mL} /$ mol at $\mathrm{T}_{\mathrm{g}}\left(-15^{\circ} \mathrm{C}\right)$ to $\sim 90 \mathrm{~mL} / \mathrm{mol}$ at $94.3^{\circ} \mathrm{C}$. For PVPh, however, neither $\tau_{\alpha}$ nor $\mathrm{m}$ can be determined experimentally. Nevertheless, Schroeder et al. ${ }^{38}$ found that fragility of poly(styrene-co-4-vinyl phenol) random copolymers was the same as measured dielectrically and mechanically. From mechanical spectroscopy, $\mathrm{m} \sim 133$ for $\tau_{\alpha}=1 \mathrm{~s} .{ }^{39}$ Using $\mathrm{dT}_{\alpha} / \mathrm{dP}=0.22 \mathrm{C} / \mathrm{MPa}$, eq 5 gives $\mathrm{V}^{\#}=560 \mathrm{~mL} / \mathrm{mol}$. This is considerably larger than the activation volume of EVA, the low- $\mathrm{T}_{\mathrm{g}}$ component, indicating the mechanism proposed by Floudas et al. ${ }^{6}$ does not apply. Of course, a hydrogen-bonded blend is fundamentally different. Whereas the components of a van der Waals mixture may relax individually, $1,2,5$ and therefore follow pressure dependences more similar to the neat components, the $\alpha$ relaxations of EVA and PVPh are coupled to form a new process in VA30 due to strong intermolecular hydrogen bonding. The dielectric segmental relaxation in this blend includes contributions from both components. For VA20, although there are two segmental processes, they are very close in frequency and cannot be cleanly resolved. The fast relaxation process (originating from unassociated EVA) dominates the dielectric loss spectra, so that defining $\tau_{\alpha}$ as the primary peak location emphasizes the contribution from unassociated EVA segments. This is confirmed by the calculated V\# for VA20 (Figure 11), 90$170 \mathrm{~mL} / \mathrm{mol}$, which is almost the same as those of neat EVA. On the other hand, $\mathrm{V} \#$ of VA30, $=\sim 160-230 \mathrm{~mL} /$ mol, is significantly larger than those for EVA but close to the composition-averaged value of PVPh and EVA.

Another possible explanation for the narrower relaxation time distributions at el evated pressure lies in the reduced intramolecular coupling of PVPh. The decrease/ weakening of hydrogen bonding with pressure occurs not only for intercomponent associations but also for the intramolecular $\mathrm{H}$-bonds in PVPh. Whereas the former leads to decoupling of segmental relaxations and broadened dielectric loss peaks, the latter would be expected to result in a reduced intramolecular friction coefficient and consequent accelerated relaxation of PVPh segments. This shifts the process to high frequencies, narrowing the apparent distribution. The observed behavior is determined by competition between these two factors. Unfortunately, although no quantitative results concerning the reduction of inter-and intramolecular hydrogen bonding with pressure are available, qualitatively the extent of intermolecular hydrogen bonding should be much higher than the intramolecular associations, at least at atmospheric pressure, considering the low PVPh concentration. Therefore, this mechanism may contribute to, but cannot fully account for, the current results.

\section{Summary}

For neat EVA, pressure and temperature play comparable roles in the segmental relaxation, as indicated by $\alpha_{\tau} /\left|\alpha_{P}\right|=2.14$, and the fact that the relaxation time distributions at different temperature-pressure conditions are essentially the same for fixed $\tau_{\alpha}$. On the other hand, temperature was found to be more important than pressure in PVPh, $\alpha_{\tau} /\left|\alpha_{P}\right|=3.35$, as a result of strong hydrogen bonding. Although the $\alpha_{\tau} /\left|\alpha_{P}\right|$ values for the miscible EVA/PVPh blends are close to that of neat EVA, a result of low PVPh concentrations (20\% and $30 \%$ ), the relaxation time distributions are narrowed with increasing pressure. This is a rather surprising observation considering (i) the large activation volume of the higher $\mathrm{T}_{g}$ PVPh compared with $\mathrm{V} \#$ of EVA, (ii) the reduction/weakening of hydrogen bonding at high temperatures and pressures, and (iii) the dominance of intermolecular hydrogen bonding. All three factors are expected to contribute to broadening of the distributions, contrary to the experimental observation. The results can be explained by considering that there are two different relaxing segments (slow $\mathrm{H}$-bonded units and fast, unassociated ones). High temperature and pressure endow the hydrogen-bonded PVPh-EVA segments with additional mobility through reducing/weakening the intermolecular interactions.

Acknowledgment. The Penn State authors express their appreciation to the National Science Foundation, Polymers Program (DM R-0211056) for support of this research. The work at NRL was supported by the Office of Naval Research.

Supporting Information Available: The log $\left(\tau_{\alpha}\right)$-volume relation, PVT measurements, and densities at constant $\tau_{\alpha}$ and constant pressure for VA20 and VA30. This material is available free of charge via the Internet at http://pubs.acs.org.

\section{References and Notes}

(1) Miller, J. B.; McGrath, K. J .; Roland, C. M.; Trask, C. A.; Garroway, A. N. Macromolecules 1990, 23, 4543.

(2) (a) Ngai, K. L.; Roland, C. M.; O'Reilly, J. M.; Sedita, J. S. Macromolecules 1992, 25, 3906. (b) Roland, C. M.; Ngai, K. L. Macromolecules 1992, 25, 363. (c) Macromol ecules 2000 33, 3184. (d) Alegria, A.; Colmenero, J .; Ngai, K. L.; Roland, C. M. Macromolecules 1994, 27, 4486.

(3) Roland, C. M.; Ngai, K. L. Macromolecules 1991, 24, 2261.

(4) Pathak, J . A. Ph.D. Thesis, The Pennsylvania State University, 2001, and references therein.

(5) Cowie, J. M. G.; Arrighi, V. In Polymer Blends and Alloys; Shonaike, G. O., Simon, G. P., Eds.; Marcel Dekker: New York, 1999; Chapter 4.

(6) Floudas, G.; Fytas, G.; Reisinger, T.; Wegner, G. J . Chem. Phys. 1999, 111, 9129.

(7) Roland, C. M.; Casalini, R. J . Chem. Phys. 2003, 119, 1838.

(8) Casalini, R.; Roland, C. M. J . Chem. Phys. 2003, 119, 4052.

(9) Alegría, A.; Gómez, D.; Colmenero, J . Macromolecules 2002, 35, 2030. 
(10) Coleman, M. M.; Graf, J . F.; Painter, P. C. Specific Interactions and the Miscibility of Polymer Blends; Technomic Publishing: Lancaster, PA, 1991.

(11) Zhang, S. H.; Painter, P. C.; Runt, J . Macromolecules 2002 35, 8478.

(12) Zhang, S. H.; Painter, P. C.; Runt, J . Macromolecules 2002, 35, 9403.

(13) The dipole moment of p-ethylphenol, a model for PVPh, is $\sim 1.6 \mathrm{D}$, while it is $\sim 1.8 \mathrm{D}$ for ethyl acetate, a small molecule analogue of the PVAc repeat unit (see ref 11).

(14) Naoki, M.; Katahira, S. J . Phys. Chem. 1991, 95, 431.

(15) Poole, P. H.; Sciortino, F.; Stanley, H. E.; Angell, C. A. Phys. Rev, Lett. 1994, 73, 1632

(16) Cook, R. E.; King, H. E.; Peiffer, D. G. Phys. Rev. Lett. 1992, 69, 3072.

(17) Ferrer, M. L.; Lawrence, C.; Demirjian, B. G.; Kivelson, D.; Alba-Simionesco, C.; Tarjus, G. J . Chem. Phys. 1998, 109, 8010.

(18) Hensel-Bielowka, S.; Paluch, M.; Roland, C. M. J. Phys. Chem. B 2002, 106, 12459.

(19) Paluch, M.; Casalini, R.; Roland, C. M. Phys. Rev. B 2002 66, 92202.

(20) Paluch, M.; Pawlus, S.; Roland, C. M. Macromolecules 2002 35,7338

(21) Paluch, M.; Roland, C. M.; Pawlus, S. J . Chem. Phys. 2002, 116, 10932.

(22) Roland, C. M.; Casalini, R.; Santangelo, P.; Sekula, M.; Ziolo, J .; Paluch, M. Macromolecules 2003, 36, 4954.

(23) Roland, C. M.; Casalini, R. Macromolecules 2003, 36, 1361.

(24) Heinrich, W.; Stoll, B. Colloid Polym. Sci. 1985, 263, 873.
(25) J ohari, G. P.; Whalley, E. Faraday Symp. Chem. Soc. 1972, $6,23$.

(26) Paluch, M.; Rzoska, S. J .; Habdas P.; Ziolo, J . J . Phys.: Condens. Matter 1996, 8, 10885

(27) Andersson, S. P.; Andersson, O. Macromolecules 1998, 31 2999.

(28) Li, G.; King, H. E.; Oliver, W. F.; Herbst, C. A.; Cummins, H. Z. Phys. Rev. Lett. 1995, 74, 2280.

(29) Roland, C. M.; Santangel o, P. G.; Ngai, K. L. J . Chem. Phys. 1999, 111, 5593.

(30) Bohmer, R.; Ngai, K. L.; Angell, C. A.; Plazek, D. J . J . Chem Phys. 1993, 99, 4201.

(31) Tait, P. G. Physics and Chemistry of the Voyage of H. M. S. Challenger; HMSO: London, 1888; Vol. 2, Part 4.

(32) Naoki, M.; Endou, H.; Matsumoto, K. J . Phys. Chem. 1987, 91,4169

(33) Paluch, M.; Roland, C. M.; Best, A. J . Chem. Phys.2002, 117, 1188.

(34) Casalini, R.; Roland, C. M. J . Chem. Phys. 2003, 119, 11951.

(35) Fontanella, J . J . J . Chem. Phys. 1999, 111, 7103.

(36) Leyser, H.; Schulte, A.; Doster, W.; Petry, W. Phys. Rev. E 1995, 51, 5899.

(37) (a) Angell, C. A. J . Non-Cryst. Solids 1991, 131-133, 13. (b). Angell, C. A. Science 1995, 267, 1924

(38) Schroeder, M. J .; Roland, C. M.; Kwei, T. K. Macromolecules 1999, 32, 6249.

(39) Santangelo, P. G., unpublished results.

MA035213Y 